\title{
Gerardo AcErenza
}

Università degli Studi di Trento, Italie

\section{Vision(s) de la littérature québécoise en Italie Révisions nécessaires?}

\begin{abstract}
Vision(s) of Quebec literature in Italy. Revisions required?
ABSTRACT: Literary texts written in French-speaking areas out of France very often represent real challenges for translators, because they have to deal with a diatopically marked language and with a large number of realia which require the implementation of specific strategies for translate in the target language. In this article, we will try to understand the strategies used by Italian translators when translating the linguistic specificities of Quebec literary works in Italian. The practice of translation can orient the vision (or reception) of a Quebec literature in Italy which appears at first sight to be hermetic because of its linguistic regionalisms. Do Italian translations of Quebec literature require revisions?
\end{abstract}

KeY wORDS: Quebec literature, translation, Italy, regionalisms, realia, revisions

\section{Introduction}

La littérature d'expression française écrite et publiée hors des frontières de l'Hexagone est très peu traduite en Italie. Seuls les textes qui remportent de prestigieux prix littéraires attirent l'attention des éditeurs italiens. Une enquête rapide menée au milieu des rayons de grandes librairies de Milan, Turin ou Bologne, montre que seul un petit nombre d'auteurs qui évoluent dans les nombreux pays de la francophonie ont été traduits et publiés en Italie. Pour ce qui est de la littérature africaine et maghrébine, les lecteurs peuvent trouver sur les rayons les traductions italiennes d'Ahmadou Kourouma, de Hampâté Ba, de Rachid Boudjedra et Yasmina Kadra. Pour ce qui est de la littérature antillaise, Maryse Condé, Patrick Chamoiseau et Raphaël Confiant ce sont les auteurs les plus traduits, mais il est parfois nécessaire de les commander auprès des libraires. 
Il est toutefois plus difficile de trouver des traductions de textes d'écrivains québécois. Comment cela s'explique-t-il? Mis à part les quelques spécialistes qui enseignent la littérature québécoise dans les universités italiennes, il est rare de s'intéresser aux romans, aux poèmes ou au théâtre québécois. Il en découle alors que la littérature québécoise n'a pas de marché en Italie et les éditeurs les plus importants ne sont pas intéressés à publier des auteurs inconnus au grand public. Même les romans d'écrivains québécois très connus en France, comme Dany Laferrière et Michel Tremblay par exemple, sont publiés en traduction par de petites maisons d'édition indépendantes à petits budgets. Dany Laferrière est publié par «La Tartaruga» de Milan, «Nottetempo » et «66thand2nd» de Rome et Michel Tremblay par la maison «Playground» de Rome. Il est par ailleurs tout à fait étonnant de découvrir à ce propos que deux romans seulement de Michel Tremblay ont été traduits et publiés en Italie: Le Cahier noir (2012) et À coeur découvert (2013).

Pourquoi donc ne traduit-on pas la littérature québécoise en Italie? S'agit-il juste d'intérêts économiques des maisons d'édition? Ou bien faut-il chercher les raisons de ce désintérêt dans la nature linguistique de ces textes, c'est-à-dire dans le caractère du français utilisé qui pose problème? Il est vrai que les textes littéraires écrits dans les aires francophones représentent très souvent de vrais défis pour les traducteurs, car ils doivent se confronter à une langue diatopiquement marquée véhiculant de plus un grand nombre de culturèmes qui nécessitent la mise en œuvre de stratégies particulières pour les rendre dans la langue cible. Dans cet article, nous tenterons de comprendre les stratégies mises en œuvre par les traducteurs italiens pour rendre dans la langue d'arrivée les spécificités linguistiques des œuvres littéraires québécoises. La pratique de la traduction peut-elle orienter la vision (ou la réception) d'une littérature étrangère qui apparaît à première vue comme hermétique à cause de sa spécificité linguistique et donc peu traduite?

\section{De la traduction des textes plurilingues}

Il existe plusieurs vérités générales sur la pratique de la traduction. Parmi les plus répandues, il convient d'évoquer celle qui présente la traduction littéraire comme une forme de passage d'un texte d'une langue-culture " $\mathrm{A}$ » à une langue-culture « $\mathrm{B}$ » et, lit-on souvent dans la littérature sur la traduction, le traducteur comme un passeur de langues, un passeur de sens et un passeur de mots. Pendant ce passage, le traducteur doit surmonter maints obstacles représentés par les particularités de la langue-culture du texte source. Pour revenir à la littérature québécoise sur laquelle nous nous concentrerons dans cette étude, la traduc- 
tion des textes d'auteurs tels que Michel Tremblay, Réjean Ducharme, Jacques Poulin et également Louis Hémon vers l'italien nécessite en effet très souvent d'une forme particulière de "négociation » (ECo 2006: ch. 14) pour tenter de restituer dans la langue de Dante toute la saveur et la couleur locales véhiculées par la langue-culture de départ. De plus, un grand nombre d'écrivains québécois pratiquent ce que Rainier Grutman a défini en termes de «hétérolinguisme littéraire », c'est-à-dire «la présence dans un texte d'idiomes étrangers, sous quelque forme que ce soit, aussi bien de variétés (sociales, régionales ou chronologiques) de la langue principale» (Grutman 1997 : 37).

Il est assez fréquent ainsi que dans les romans, dans les nouvelles ou dans les pièces de théâtre de la littérature québécoise, se croisent et se superposent plusieurs langues et plusieurs niveaux de langue. Que l'on pense en particulier à la difficulté de traduire les textes qui appartiennent à la littérature québécoise «joualisante» des années soixante et soixante-dix. Ces textes se caractérisent par une forme particulière d'hybridité linguistique typique de ce que l'on a désigné pendant longtemps comme le parler «joual». Puisqu'il n'existe pas dans la culture de la langue d'arrivée un code qui soit l'équivalent de ce parler, comment règle-t-on les problèmes de traduction que de tels textes posent-ils? La traduction italienne des Belles-Sœurs de Michel Tremblay (1994) par exemple, permet-elle de restituer les mêmes fonctions subversive, identitaire et esthétique que le «joual» véhicule dans la pièce? Et de quelle manière? Bref, une traduction d'une œuvre littéraire québécoise, pour ce qui est de la langue-culture, a quelque chose de québécois à offrir aux lecteurs italiens?

Depuis que nous étudions les traductions italiennes d'œuvres appartenant à la littérature québécoise, nous avons pu constater qu'il existe une tendance généralisée des traducteurs qui vise à standardiser, à normaliser la langue-culture $\mathrm{du}$ texte source. Toutefois, selon David Bellos, traductologue étasunien de Princeton,

l'approche consistant à domestiquer et acclimater dans la traduction [...] a pu être dénoncée par certains critiques comme étant une marque de 'violence ethnocentrique'. Selon eux, l'éthique de la traduction impose aux traducteurs de ne pas éradiquer toute l'étrangeté, ou pour mieux dire 'l'étrangèreté', d'un texte écrit dans une autre langue.

BeLLOS 2012: 53

Parfois, toujours selon Bellos, cette "étrangèreté » est considérée par certains - qu'il ne désigne pas - comme une maladresse de style ou bien comme une erreur du traducteur. Or, la question qui s'impose est la suivante: l'«étrangèreté» de l'original est-elle rendue dans les traductions italiennes des textes québécois? Comment les traits linguistiques et culturels du texte source sont-ils transposés en italien? 
Nous nous proposons d'apporter des réponses à ces questions en analysant les traductions italiennes de quelques classiques et de quelques textes contemporains de la littérature québécoise. Tout au long de notre parcours, nous convoquerons d'un côté la théorie des «tendances déformantes» du traductologue Antoine Berman et de l'autre nous ferons également référence aux réflexions sur la traduction des variétés linguistiques, c'est-à-dire sur la traduction des «sociolectes» et des parlers vernaculaires, proposées dans les contributions du volume 7 de la revue TTR (1994). Dans le texte de présentation du volume, Annick Chapdelaine et Gillian Lane-Mercier soulignent en effet que les «sociolectes sont susceptibles d'être étudiés sur le double plan de la forme et du contenu» (1994: 7). Pour ce qui est de la «forme», ils permettent tout d'abord de «tenir compte des prononciations, des constructions phrastiques, des lexèmes et des expressions », tandis que pour ce qui est du «contenu», ils permettent de «rendre compte des valeurs, des croyances, des constructions identitaires, des compétences et des pratiques qui, connotés par l'emploi de telle unité linguistique non standard, signalent l'appartenance à un (sous-)groupe précis » (1994: 7). Nous allons nous référer à ces cadres théoriques afin de voir si le tissu signifiant que représente la présence de la variété québécoise dans des textes littéraires, avec ses régionalismes, ses anglicismes et l'oralité typiques, est transposé dans les traductions italiennes et de quelle manière.

Dès la publication de son premier ouvrage intitulé L'Épreuve de l'étranger (1984), Antoine Berman condamne la traduction ethnocentrique et la négation méthodique de "l'étrangèreté» de l'œuvre originale et souhaite une réflexion « éthique» pour ce qui est de l'acte du traduire. Dans La traduction et la Lettre ou l'Auberge du lointain (1999), il revendique que l'on opère des «traductions à la Lettre», ce qui ne signifie pas une traduction mot à mot, mais plutôt une traduction qui respecte la «fidélité à la Lettre» et non seulement pas au «sens», une attention spécifique au «particulier» au détriment de ce qu'il appelle «l'universel», une attention à toutes «les dimensions auxquelles s'attaque le système des déformations» (1999: 67). Pour lui, «la fidélité au sens s'oppose [...] à la fidélité à la Lettre» (1999: 34).

Antoine BERMAN se révolte en effet contre la tendance généralisée des traductions ethnocentriques, c'est-à-dire ces traductions qui ramènent tout aux normes, aux valeurs de la culture d'arrivée et qui considèrent l'étranger comme un intrus qui doit être «annexé, adapté, pour accroître la richesse de cette culture» (1999: 29). Il en découle que «toute trace de la langue d'origine doit avoir disparue, ou être délimitée, la traduction doit être écrite dans une langue normative, elle ne doit pas heurter par des 'étrangetés' lexicales ou syntaxiques » (1999: 35). Il sollicite donc une «visée éthique du traduire» qui «propose d'accueillir l'étranger dans sa corporéité charnelle» et cet accueil ne peut se réaliser que grâce à la « fidélité à la Lettre de l'œuvre» (1999: 77) : 
Le traducteur qui traduit pour le public est amené à trahir l'original, à lui préférer son public, qu'il ne trahit d'ailleurs pas moins, puisqu'il lui présente une œuvre «arrangée» [...]. Amender une œuvre de ses étrangetés pour faciliter sa lecture n'aboutit qu'à la défigurer et, donc, à tromper le lecteur que l'on prétend servir. Il faut bien plutôt [...] une éducation à l'étrangeté.

BERMAN 1999: 71-72

En privilégiant la «belle forme» et la seule transmission du «sens», les traducteurs détruisent systématiquement la «lettre des originaux» (1999: 52) par le moyen de transformations que Berman appelle «tendances déformantes». Il en théorise treize: «la rationalisation, la clarification, l'allongement, l'ennoblissement, l'appauvrissement quantitatif et qualitatif, l'homogénéisation, la destruction des rythmes, la destruction des réseaux signifiants sous-jacents, la destruction des systématismes, la destruction des réseaux langagiers vernaculaires, la destruction des locutions et effacements de superposition de langues » (1999: 53).

Les limites imposées à cet article ne nous permettent pas de détailler chacune de ces treize «tendances déformantes» théorisées par Berman, mais nous le ferons pour quelques-unes d'entre elles lors de la comparaison et du commentaire critique des traductions que nous avons analysées pour cette étude.

De la même manière, Bernard Vidal souligne, dans une étude qui porte sur les traductions françaises du "vernaculaire noir américain », que les traducteurs français «trop à l'écoute de [leur] culture» choisissent d'habitude «le parti pris [...] de la neutralisation du dialecte noir en conformant [leurs] ressources aux seules limites de l'Hexagone». À l'instar d'Antoine Berman, Bernard Vidal ajoute que

de tels choix constituent une fausse représentation ; qu'ils neutralisent en les déviant les enjeux engagés par les écrivains dans leur langue d'origine ; qu'ils entérinent une fois de plus la conception de l'étranger comme un lieu qu'il convient de rapatrier, dans l'acte de traduction, par l'effacement de toute distance culturelle et la promotion de l'identité.

VIDAL $1994: 168$

Le même constat est fait par Marion Beaujard pour ce qui est des stratégies de traduction de l'anglo-irlandais en français. En étudiant la traduction française du roman A Star Called Henry de l'écrivain irlandais Roddy Doyle, elle souligne à plusieurs reprises que «les formes régionalement marquées du texte d'origine disparaissent dans le texte traduit, lequel présente une langue parfaitement standardisée. [...] Tous les éléments régionaux disparaissent en français, rien ne dévie des formes standardisées» (BEAUJARD 2013 : 137). En ce qui a trait au lexique «typiquement irlandais» du texte source, «composé de variantes phonétiques, d'emprunts directs ou anglicisés au gaélique, ou encore de survivance 
d'anciennes formes de l'anglais», BEAUJARD remarque que «le traducteur a fait le choix de la standardisation, ne traduisant que le contenu sémantique et laissant la forme régionale de côté» (2013: 134-135).

Qu'il s'agisse d'une langue de «culture » (Berman / Bellos), du vernaculaire noir américain (Vidal) ou de l'anglo-irlandais (Beaujard), force est de constater que les traducteurs choisissent de standardiser toute forme de variation diatopique ou diastratique présente dans la langue du texte source. Qu'en estil des stratégies mises en œuvre par les traducteurs italiens de la littérature québécoise?

\section{Vision(s) de la littérature québécoise traduite en italien}

Pour entrer dans le vif du sujet, nous commencerons par évoquer la traduction italienne de la pièce Les Belles-Sours de Michel Tremblay (1968) en soulignant certains problèmes qu'elle présente. Les traducteurs de cette pièce ont choisi un niveau de la langue d'arrivée proche du standard italien en normalisant tous les différents niveaux de langue propres à chaque personnage. Si l'on compare, en guise d'exemple, certaines répliques de Germaine Lauzon et de Lisette de Courval, deux personnages de la pièce que l'on peut considérer comme antagonistes d'un point de vue linguistique, on remarque que dans la version italienne la langue de ces deux personnages ne présente aucune caractérisation, tandis que dans le texte de départ leur registre linguistique est différemment marqué :

Germaine Lauzon: Tu pourrais pas r'mettre ça à demain? J'ai une idée, 'coute ben... À midi, j'ai téléphoné à mes sœurs, à la sœur de ton père, pis chus t'allée voir les voisines.

Tremblay 1968: 16

Lisette de Courval: Mon Dieu, que vous êtes donc mal embouchée, madame Brouillette! Regardez, moi, j’perle bien, puis je m'en sens pas plus mal!

Tremblay 1968: 25

Germaine Lauzon: Non puoi rimandare a domani? Perché ho avuto un'idea, ascolta... A mezzogiorno ho chiamato le mie sorelle, la sorella di tuo padre e sono andata dalle vicine.

Tremblay $1994: 28$

Lisette de Courval: Dio mio, come è sboccata, signora Brouillette! Non costa poi tanto parlare perbene, guardi me! 
La différence des niveaux de langue de ces deux personnages, qui est l'un des motifs importants de la pièce, puisque Lisette de Courval est le seul personnage qui a voyagé en France, «en Urope» comme elle dit, et se targue de bien «perler» (25), avec de nombreux phénomènes d'hypercorrection, n'est pas rendue dans la version italienne où les traducteurs Francesca Moccagatta et Jean-René Lemoine effacent cette opposition linguistique qui vise à ridiculiser la manière pointue de parler le français de France. Le texte d'arrivée ne peut pas véhiculer les mêmes fonctions du texte de départ et l'identité québécoise du texte est aplatie. L'opposition oralité-standard n'est pas du tout rendue. En tant que lecteur bilingue, on ne ressent pas le même plaisir du texte. Ce n'est qu'un exemple de la difficulté que les traducteurs doivent surmonter lorsqu'un doit traduire une langue connotée comme le français des personnages de la pièce de Michel Tremblay. La normalisation, avec l'effacement des niveaux et de la superposition des langues, semble être la stratégie la plus récurrente. Y aurait-il d'autres stratégies pour mieux rendre la complexité de cette langue en italien? Nous y reviendrons dans la dernière partie de cette étude.

Dans les trois exemples suivants, tirés de la traduction italienne de Volkswagen blues de Jacques Poulin, on peut noter comment la traductrice Maria Rosa Baldi, qui traduit pour la petite maison d'édition de Rome appelée «Hortus Conclusus », élimine la superposition des langues française et anglaise :

Il dit excusez-moi et il se dirige vers le comptoir. Il emprunte un stylo à la barmaid.

Poulin 1989: 47

Dice «Scusate» e si dirige verso il banco. Prende a prestito una penna dalla barista.

Poulin $2000: 45$

Ce qu'il aimait, c'étaient les voyages, les autos. Il faisait des petites jobs et quand il avait un peu d'argent, il partait en voyage.

Poulin 1989: 13

Gli piacevano i viaggi, le auto. Faceva dei lavoretti e quando aveva un po' di soldi si metteva in viaggio.

Poulin 2000 : 10

C'était mon plus grand chum autrefois [...].

Poulin 1989: 14

Una volta era il mio migliore amico [...].

Poulin 2000: 12

Antoine Berman souligne à ce propos qu'il s'agit «peut-être du problème le plus aigu que pose la traduction de la prose, car toute prose se caractérise par des superpositions de langues plus ou moins déclarées » (1999: 66). Dans les exemples ci-dessus, la superposition des langues est explicitement déclarée et 
la présence de l'anglais dans les trois répliques caractérise la langue du texte, car l'auteur n'hésite pas à utiliser des anglicismes assez fréquents au Québec. Dans ce roman en particulier, l'anglais, avec le français, l'italien et l'allemand est l'une des langues du roman et joue un rôle important dans le tissu signifiant du texte. Bien qu'elle traduise les nombreuses répliques des personnages en anglais en notes du traducteur placées à la fin du texte, la traductrice normalise tous les anglicismes qui se trouvent dans les phrases françaises en éliminant ainsi des traits caractéristiques des locuteurs québécois tant réels que fictionnels.

Dans l'exemple qui suit, la traductrice italienne a eu besoin de cinq mots pour rendre le terme "Pinkerton» qui désigne un agent de sécurité dans les magasins nord-américains...

Un Pinkerton se tenait à l'entrée, près du comptoir [...].

Poulin 1989: 69

All'entrata, vicino al banco, c'era un agente del servizio di guardia [...].

Poulin 2000: 64

La clarification est une tendance déformante très fréquente qui a pour but de «rendre clair ce qui ne l'est pas et ne veut pas l'être dans l'original» (BERMAN 1999: 55), mais qui allonge de surcroît le texte cible par rapport au texte source. Selon Antoine Berman, toute traduction est plus longue que l'original et il précise que l'allongement quantitatif est une conséquence directe de la rationalisation et de la clarification.

Une autre tendance déformante que l'on peut fréquemment observer chez les traducteurs italiens de la littérature québécoise est la destruction des réseaux vernaculaires. Selon Antoine Berman, «toute grande prose entretient des rapports étroits avec les langues vernaculaires [...]. L'effacement des vernaculaires est une grave atteinte à la textualité des œuvres en prose» (1999: 64).

Parfois, l'effacement des vernaculaires est une conséquence directe des compétences du traducteur qui manifeste indirectement des limites à la compréhension du texte de départ. En effet, l'un des problèmes majeurs pour le traducteur aux prises avec la littérature québécoise est la reconnaissance des régionalismes du français québécois. Par conséquent, nous avons l'impression que Francesco Gazzé, qui a traduit Nikolski de Nicolas Dickner, n’a jamais voyagé au Québec, ou à Montréal, car il traduit le mot «dépanneur» selon l'acception française de «garagiste» (en italien meccanico) et non pas selon l'acception québécoise de petit magasin de quartier.

- ¡Claro!, s'esclaffe le gérant en lui montrant un rutilant CCM 1977 modifié, stationné devant la vitrine du dépanneur. 
- ¡Claro!, scoppia a ridere il gestore, mostrandogli una fiammeggiante CCM 1977 modificata, parcheggiata davanti alla vetrina del meccanico.

DICKNER 2008: 77

Or, tous les touristes qui visitent Montréal ou le Québec savent qu'un « dépanneur» n'est pas un «garagiste», mais un petit commerce qui ouvre très tôt le matin et ferme très tard le soir et qui «dépanne» avec toutes sortes de produits de consommation. Le sens du texte d'arrivée n'est pas trop trahi, toutefois l'esprit de la «Lettre », la fidélité «à la Lettre» selon l'acception bermanienne oui... De plus, en empruntant la réflexion que Marion BEAUJARD fait sur la traduction des termes gaéliques en français, nous pouvons dire à notre tour que le mot italien meccanico ne véhicule pas la même «nuance culturelle » évoquée dans le texte de départ et que la solution choisie par le traducteur italien ne fonctionne ni comme «vecteur d'étrangéité », ni comme «facteur d'identification» (2013 : 134). Bref, on ne retrouve pas la présence linguistique signalant l'appartenance à un «(sous-)groupe précis» (CHAPDELAINE et LANE-MERCIER 1994: 7).

La même remarque doit être faite pour la traduction normalisante de Cristiano Felice du régionalisme «débarbouillette» qui figure dans une nouvelle de Nadine Bismuth:

[...] pour ne pas abîmer le tissu soyeux, il faut prendre une débarbouillette.

Bismuth 1999: 25

[...] per non rovinare la seta devo prendere un piccolo asciugamano.

BISMUTH 2003 : 16

La «débarbouillette» qui «n'est pas en usage en France, où l'on utilise le 'gant de toilette' et inversement, le 'gant de toilette' est inconnu au Québec» (Menay 2003 : 625) est en effet un carré de tissu éponge qui remplace le gant de toilette. Le traducteur italien traduit ce terme en recourant à l'hyperonyme «petite serviette» (en italien piccolo asciugamano) et normalise ainsi un régionalisme du français parlé au Québec. Toutefois, la solution proposée n'est pas satisfaisante puisqu'il existe en italien l'équivalent guanto da bagno.

Cependant, ce qui frappe l'analyste qui compare la version italienne des nouvelles de Nadine Bismuth avec le texte en français, c'est la stratégie utilisée pour la traduction des nombreux «sacres» prononcés par les personnages de l'écrivaine québécoise. Le traducteur Cristiano Felice les rend presque tous en italien en utilisant un seul terme qui appartient au domaine scatologique. Ainsi, dans les exemples suivants, les sacres «tabarnak», " crisse » et " hostie/ostie », ont tous été rendus avec l'interjection cazzo, terme scatologique qui désigne en italien de manière familière et vulgaire le sexe masculin. Et de plus, le traducteur a choisi la même interjection italienne pour rendre l'interjection anglaise bullshit... 
[...] ça me donne des migraines, l'encens, tabarnak, ça me rappelle quand j'étais jeune $[\ldots]$.

Bismuth 1999: 38

[...] l'incenso, cazzo, fa venire il mal di testa, mi ricorda quando da giovane [...]. Bismuth 2003 : 24-25

[...] je me suis tournée de mon côté : "Crisse que t'es cheap" lui ai-je dit [...]. Bismuth 1999: 57

[...] mi sono girata dall'altra parte. "Cazzo, ma sei proprio un poveraccio!" ho detto $[\ldots]$

BISMUTH $2003: 36$

T'hallucines des morpions partout et t'obsèdes sur une ostie de paire de gougounes $[\ldots]$.

BISMUth 1999: 141

Vedi piattole dappertutto e ti fissi per comprare un paio di ciabatte del cazzo [...]. Bismuth 2003 : 91

Site historique, site historique, bullshit, ouais, a-t-il grogné [...].

Bismuth 1999: 155

Sito storico, sì, proprio un sito storico del cazzo! ha brontolato [...].

Or, ces sacres (jurons) que Nadine Bismuth utilise dans ses nouvelles font partie intégrante du vocabulaire québécois parlé et font référence aux objets sacrés et à la religion qui a occupé une place centrale au Québec dans les années soixante. Le clergé a longtemps exercé une très forte influence sur la société et les Québécois, par sentiment de révolte contre le contrôle excessif de l'Église sur les mœurs de l'époque, ont commencé à s'attaquer aux objets qui représentaient indirectement ce contrôle. L'utilisation des sacres permettait ainsi de contester l'autorité de la religion catholique exercée sur toutes les couches de la société québécoise. Les jurons québécois, comme le vernaculaire noir américain, sont donc «porteurs de sens, de messages» et si on les remplace dans la langue cible avec des termes appartenant au domaine scatologique, «les sens» et les «messages » sont perdus de manière considérable (LAVOIE 1994 : 124).

Utilisé comme une interjection dans le premier exemple, le sacre «tabarnak» vient de «tabarnacle» qui est un objet liturgique et l'orthographe varie souvent. «Crisse » est la variante oralisée de "Crist» et dans le deuxième exemple ce sacre est également utilisé comme une interjection. Tandis que le juron «ostie/ hostie » est utilisé dans le troisième exemple comme un adjectif qualificatif et son origine religieuse est aussi évidente.

Comment traduire ces sacres sans trahir «la Lettre» du texte source? Les sacres représentent une vraie particularité de l'oralité québécoise. La stratégie du traducteur italien Cristiano Felice n'est pas vraiment réussie, car en utilisant 
la même interjection scatologique italienne (cazzo!) il appauvrit qualitativement le texte. Cette tendance, selon Berman, consiste à remplacer «des termes, expressions, tournures, etc., de l'original par des termes, expressions, tournures, n'ayant ni leur richesse sonore, ni leur richesse signifiante ou - mieux - iconique » (1999: 58).

Avec les régionalismes, les culturèmes également représentent de vrais obstacles pour les traducteurs européens de la littérature québécoise. Il semble que la traductrice de Volkswagen blues Maria Rosa Baldi n'a pas vraiment compris la nature du "pâté chinois», un mets typiquement québécois qui n’a rien de... chinois. Il s'agit d'un plat traditionnel au Québec "fait de bœuf haché, de pomme de terre en purée et de grains de maïs» qui est souvent comparé à un hachis Parmentier sans maïs. Selon Lionel Menay, le "pâté chinois » est «ainsi appelé parce qu'on le servait aux ouvriers chinois qui construisaient les lignes de chemin de fer au Canada» (2003: 1264). Toutefois, selon une autre version de l'origine de ce mets aujourd'hui encore très populaire au Québec, il s'agirait de la "Chine pie», une spécialité de la ville de China, dans le Maine, aux États-Unis, où il y avait des émigrés canadiens-français. Répétons-le, dans le "pâté chinois » québécois il n'y a rien de chinois, tandis que selon la traductrice italienne cela serait un "pâté » qui vient ou qui est typique de Chine.

\section{Un pâté chinois. \\ Poulin 1989: 155 \\ Un paté cinese. \\ PouLIN 2000: 144}

La traductrice a senti le besoin de placer à la fin du texte 96 notes du traducteur, surtout pour traduire plusieurs répliques très faciles en anglais du texte source, mais elle aurait pu bien en ajouter une pour expliciter un aspect typique de la cuisine québécoise, pour offrir quelque chose de typiquement québécois aux lecteurs italiens.

Cristiano Felice, le traducteur italien des nouvelles de Nadine Bismuth, a également eu des problèmes pour la traduction de ce passage où le personnage évoque une réalité bien québécoise :

Le chocolat, c'est bien bon, mais la vanille avec le temps des cabanes à sucre qui s'en vient, c'est si délicieux avec de la tire d'érable étendue dessus.

Bismuth 1999: 174-175

Il cioccolato è buonissimo ma la vaniglia, adesso che è quasi arrivata la stagione, è deliziosa spalmata con lo sciroppo d'acero. 
Or, «la cabane à sucre» et la «tire d'érable» sont des éléments typiques de la langue-culture du Québec et n'ont pas d'équivalents dans la langue-culture d'arrivée. Toutefois, le traducteur aurait pu trouver une autre manière pour ne pas éliminer totalement le rendez-vous traditionnel des Québécois avec la «cabane à sucre», un événement qui marque la fin du long hiver et l'arrivée du printemps. La stratégie utilisée appauvrit considérablement le texte d'arrivée puisqu'il évoque seulement l'arrivée du printemps (en italien stagione).

Nous avons déjà analysé dans une autre étude les traductions italiennes de Maria Chapdelaine de Louis Hémon (ACERENZa 2011). Toutefois, nous venons de découvrir une version de ce chef-d'œuvre «réduite et annotée à l'usage des écoles italiennes par Alceste Bisi». Le texte est intégralement en français, mais il y a un grand nombre de notes de bas pages (4-5 notes par page en moyenne) dans lesquelles la traductrice propose une traduction italienne des mots les plus difficiles et surtout... des nombreux canadianismes qui se trouvent dans le texte. Il s'agit d'une publication de 1967 (Hémon) et en réalité cela serait la meilleure manière de faire découvrir à de jeunes étudiants italiens un texte si important de la littérature québécoise.

Cependant, ces notes du traducteur placées en bas de page trahissent parfois le vrai sens des canadianismes, car la traductrice n'a pas vraiment compris la nature de l'objet désigné. Par exemple, le terme anglais «foreman de chantier», qui désigne un "contremaître», un "chef d'équipe» où un «chef de chantier» comme dans Maria Chapdelaine, a été traduit par la paraphrase chi vuole arruolarsi (HÉMON 1967: 4) et le verbe pronominal italien arruolarsi signifie plutôt «s'engager», ou bien «s'enrôler, recruter». L'expression de «l'eau frette» (41) a été traduite par l'expression italienne acqua fresca (de l'eau fraîche), tandis que l'adjectif «frette» en français québécois est utilisé pour qualifier quelque chose qui est plus froid que le froid ordinaire, qui est «glacial» comme le souligne Lionel Menay dans son Dictionnaire québécois-français (2003: 869).

Comme l'avaient déjà fait les autres traducteurs italiens de Maria Chapdelaine, la traduction des termes «bleuet» et «atoca», régionalismes du français québécois qui désignent respectivement la «myrtille» et la «canneberge » a posé d'énormes problèmes à Alceste Bisi, car elle/il a traduit le terme «bleuet»par mora (mûre) et «atoca» par l'hyperonyme bacca (baie). Il est vrai qu'en 1967, il était sans doute plus difficile d'avoir accès à des dictionnaires du français québécois, mais cela montre quand même la difficulté que les traducteurs de la littérature québécoise ont rencontrée et rencontrent encore de nos jours.

La normalisation de ces caractéristiques lexicales est-elle alors la seule stratégie envisageable? Ou bien y aurait-il besoin d'une révision, d'une autre manière de garder «l'étrangèreté » du texte de départ? Si la traductrice de cette version réduite de Maria Chapdelaine décide de traduire le mot «drave» par segheria (en français scierie) peut-on la blâmer éthiquement? Commet-elle une espèce de « violence ethnocentrique» en effaçant du texte cible le caractère «étranger» du 
texte source et en généralisant un trait important de la langue-culture de départ? On sait que la segheria (la scierie) est l'étape suivante à la «drave» qui consiste à transporter des billots de bois par le courant d'un fleuve sous le contrôle des «draveurs» qui évitent aux billots de se bloquer pendant le flottage vers les ports fluviaux.

\section{Conclusion}

En guise de conclusion, nous aimerions évoquer une expérience didactique relative à la traduction des Belles-Sœurs de Michel Tremblay. Lors d'un cours de théorie et pratique de la traduction littéraire, nous avons demandé à nos étudiants de traduire des passages de la pièce de leur choix en utilisant le dialecte qu'ils utilisent au quotidien à la maison ou avec les amis. Chaque étudiant du cours a ainsi traduit dans son propre dialecte plusieurs répliques de la pièce et le résultat est assez intéressant par rapport à la version aseptisée proposée par les traducteurs Moccagatta et Lemoine.

Voici des extraits en dialecte vénitien de la ville de Vicenza traduit par l'étudiante Giorgia Rigoni (1), en dialecte des Marches de la ville d'Ancona traduit par l'étudiante Martina Belelli (2) et en dialecte lombard de la ville de Brescia traduit par Marta Camilla Fogli (3) :

(1) Germana - O no? Anca mi so restà sorpresa! Stamattina te geri pena partia e i ga sonà el campaneo! Vo vedere chi che xe. Gera un tosato! El te saria piaxudo, gera proprio el to tipo. El gavarà vudo ventido, ventitré ani, cavej neri, rissi, bafeti... Proprio un bel omo! El me domanda se son la signora Germana Lauzon, casalinga. A ghe go dito, certo che so mi. El me ga dito che gera i me bolini. Te po maearte, gero tutta agità. No savevo co dire... Do tosi li ga portà dentro casa e che l'altro che parlava... Te sentissi come chel parlava ben! L'era posà! Varda, so sicura chel te saria piaxudo, Linda...

(2) Germaine Lauzon: A posto, no? So rimasta sorpresa pure io! Eri partita da pogo, sta mattina e ha sonato un Cristo alto. El potevi amà, Linda, el tipo tua, C'avrà avuto ventidò o ventitré anni, capelli neri, ricciulì, co un poghetto de baffi... propio un bel'omo. Ha chiesto si c'era la signora Germaine Lauzon ho detto che so io. Lu m'ha detto che so i timbri mia. Ero nervosa da morì, me capisci sci? Nun sapevo cu dì. Du ragazzi enne venuti a portalli a casa, po l'altro ragazzo m'ha fatto na specie de discorso... Parlava bè, chiedeva per favore! Era fine, so scigura che te poteva piacé, Linda...

(3) Germana Lanzini: Era? Ma ha l'aspetae gne me! Ta heret apena andanda de fo stamatina, che i ga hunat a la porta, andò a dervi, l'era an scheturlot. Per 
me l'era jù che ta piasia Lindo. Propre el to gener. Intedù, intetré ain, caei negher, rihuli, coi bafeti... propre en bel om. El ma dumanda si here la sciura Germana Lanzini, casalingo. Ga dise de he, che ho me. Lu 'l dis, go che i ho bulì. M'ennit n'agitasiù. El here pio coho dì. Do schet ie 'gnit a purtai dan la cà e po' l'oter schet m'ha fat an discur... el parlao come 'n shor! L'era fine! Te Linda, l'ares truat de to gusto.

À partir de cette expérience de pratique de la traduction littéraire avec nos étudiantes, nous avons pu tirer plusieurs conclusions dont la principale a été que les variétés dialectales italiennes se prêteraient mieux à la traduction du français des Belles-Sœurs de Michel Tremblay, car elles permettent de créer un décalage avec le standard italien et le succès de la version écossaise des Belles-Sœurs confirme notre impression.

Il faut toutefois préciser qu'il s'agit d'une stratégie efficace dans le cadre d'une expérience didactique et que Berman en particulier est assez critique lorsqu'on rend une variété régionale de la langue source par une autre variété régionale dans la langue cible: «Malheureusement, le vernaculaire ne peut être traduit dans un autre vernaculaire. Seules les koinai, les langues 'cultivées' peuvent s'entre-traduire. Une telle exotisation, qui rend l'étranger du dehors par celui du dedans, n'aboutit qu'à ridiculiser l'original» (BERMAN 1999 : 64).

\section{Bibliographie}

ACERenZA, Gerardo, 2001: "Les canadianismes, ces inconnus: les traductions italiennes de Maria Chapdelaine de Louis Hémon». Études de Linguistique Appliquée, nº 4/164, pp. $405-420$.

Beaujard, Marion, 2013: «Les stratégies de traduction de la langue anglo-irlandaise dans A Star Called Henry de Roddy Doyle ». Palimpsestes, n 26, pp. 131-149.

Bellos, David, 2012: Le poisson et le bananier. Une histoire fabuleuse de la traduction. Trad. de D. LOAYZA avec la collaboration de l'auteur. Paris, Flammarion.

Berman, Antoine, 1984: L'Épreuve de l'étranger. Culture et traduction dans l'Allemagne romantique. Paris, Gallimard, coll. «Tel».

Berman, Antoine, 1999: La traduction et la Lettre ou l'Auberge du lointain. Paris, Seuil, coll. «L'Ordre philosophique » [1985].

Bismuth, Nadine, 1999: Les gens fidèles ne font pas les nouvelles. Montréal, Boréal.

Bismuth, Nadine, 2001: La fedeltà non fa notizia. Trad. C. Felice. Roma, Voland, coll. «Le Amazzoni».

Chapdelaine, Annick, Lane-Mercier, Gillian, 1994: «Présentation: traduire les sociolectes: définitions, problématiques, enjeux ». TTR: traduction, terminologie, rédaction, vol. $7, \mathrm{n}^{\circ} 2$, pp. 7-10.

Dickner, Nicolas, 2007 : Nikolski. Montréal, Alto.

Dickner, Nicolas, 2008 : Nikolski. Trad. Francesco Gazzè. Roma, Voland. 
Eco, Umberto, 2006 : Dire presque la même chose. Expériences de traduction. Trad. l'italien par M. Bouzaher. Paris, Éditions Grasset et Fasquelle.

Grutman, Rainier, 1997: Des langues qui résonnent. L'hétérolinguisme au XIX siècle québécois. Montréal, Fides/Cétuq.

HÉmon, Louis, 1967 : Maria Chapdelaine. Récit du Canada français. Trad., introd. e note di Alceste BisI. Roma, Società Ed. Dante Alighieri, coll. «Autori stranieri annotati per le scuole italiane».

Lavoie, Judith, 1994: «Problème de traduction du vernaculaire noir américain: le cas de The Adventures of Huckleberry Finn ». TTR: traduction, terminologie, rédaction, vol. 7, $\mathrm{n}^{\circ} 2$, pp. $115-145$.

Menay, Lionel, 2003 : Dictionnaire québécois-français. Montréal, Guérin.

Poulin, Jacques, 1989: Volkswagen Blues. Montréal, Québec/Amérique.

Poulin, Jacques, 2000 : Volkswagen Blues. Trad. Maria Rosa BALDI. Roma, Hortus Conclusus.

Tremblay, Michel, 1968: Les Belles-Sœurs. Montréal, Leméac.

Tremblay, Michel, 1994 : Le cognate. Trad. F. Moccagatta et J.-R. Lemoine. In: Teatro del Québec. Ubulibri, Milano.

Tremblay, Michel, 2012: Il quadreno nero. Trad. L. Di Lella et M.L. Vanorio. Roma, Playground.

Tremblay, Michel, 2013 : Il cuore a nudo. Trad. F. Di Lella e L. Di Lella. Roma, Playground.

VIDAL, Bernard, 1994: "Le vernaculaire noir américain: ses enjeux pour la traduction envisagés à travers deux œuvres d'écrivaines noires, Zora Neale Hurston et Alice Walker ». TTR: traduction, terminologie, rédaction, vol. 7, $\mathrm{n}^{\circ}$ 2, pp. 165-207.

\section{Notice biographique}

Gerardo Acerenza est professeur associé de Langue et Traduction françaises au Département des Lettres et Philosophie de l'Università degli Studi di Trento (Italie). Il a publié plusieurs articles sur le débat linguistique au Québec, sur la traduction des canadianismes en italien et sur l'œuvre de l'écrivain québécois Jacques Ferron (Des voix superposées : plurilinguisme, polyphonie et hybridation langagière dans l'œuvre romanesque de Jacques Ferron, Trento, Università degli Studi di Trento - Dipartimento di Studi Letterari, Linguistici e Filologici, coll. «Labirinti», 2010). En décembre 2017, il a conçu et organisé un colloque international ayant pour titre «Qu'est-ce qu'une mauvaise traduction littéraire?» qui a eu lieu au Département des Lettres et Philosophie de l'Université de Trento en Italie.

gerardo.acerenza@unitn.it 